\title{
A prospective randomized, placebo-controlled trial comparing mifepristone and vaginal misoprostol to vaginal misoprostol alone for elective termination of early pregnancy
}

\author{
Roopa Malik*, Viral Kumar, Vijayata Sangwan, Smiti Nanda
}

Post Graduate Institute of Medical Science, Rohtak, India

Email: ${ }^{*}$ drroopa.sangwan@gmail.com

Received 1 October 2011; revised 8 November 2011; accepted 10 December 2011

\begin{abstract}
A prospective randomized, placebo-controlled trial comparing mifepristone and vaginal misoprostol to vaginal misoprostol alone for elective termination of early pregnancy. Author: Dr Roopa Malik, Assistant Professor, Obstetrics and gynaecology department Pt BDS PGIMS Rohtak. Background: Vaginal misoprostol has been shown to be an effective single agent for medical agent for medical abortion. This randomized, placebo controlled trial compared a regimen of mefipristone and misoprostol with misoprostol alone for termination of early pregnancy. Methods: 200 women with gestation < 56 days were randomized by a random number table to receive either $200 \mathrm{mg}$ mifepristone orally or placebo followed 48 h later by 800 ug vaginal misoprostol. Abortion success was defined as complete abortion without the use of surgical aspiration. Results: Successful medical abortions occurred in 96 out of 100 subjects $(96 \%)$ after mifepristone followed by vaginal misoprostol. In all, 79 out of 100 subjects (79\%) successfully aborted after placebo and vaginal misoprostol. The higher success rate of complete abortion with mifepristone and misoprostol regimen was statistically significant compared with the placebo and misoprostol regimen $(p<0.05)$. Conclusion: A regimen of mifepristone and misoprostol was significantly more effective for termination of pregnancies < 56 days than misoprostol alone. The misoprostol alone regimen for termination of early pregnancy is not a very good method for medical abortion but $79 \%$ efficacy obtained with vaginal misoprostol alone may clinically acceptable when mifepristone is not available.
\end{abstract}

${ }^{*}$ Corresponding author.
Keywords: Abortion; Medical Abortion; Mifepristone; Misoprostol; Pregnancy

\section{INTRODUCTION}

Termination of pregnancy is a medically directed miscarriage prior to independent viability, using pharmacological or surgical means. It is estimated that 46 million pregnancies are terminated voluntarily each year, 27 million carried out under safe conditions and 19 million falling into the category of "unsafe abortions" [1]. Until the second half of twentieth century, dilatation and curettage (D \& C) was the most common and virtually only method used for safe abortion.

Abortion by vaccum aspiration gained acceptance in the 1960s and has become the standard of care. However, being a surgical technique, it is associated with certain risks such as perforation of uterus, haemorrhage and cervical laceration. The late complications include infection, retained products, thromboembolic phenomenon, anaemia and often subsequent infertility due to uterine synechiae formation. The average incidence of complications is $1 \%$ and of failure is $0.5 \%$ [2].

Pregnancy can also be terminated safely pharmacologically. The main advantage of medical abortion is that it allows women to avoid the risks of surgery and anaesthesia. A regimen of $600 \mathrm{mg}$ of mifepristone followed by 400 ug oral misoprostol was approved for use in USA in September 2000 for elective termination of pregnancy up to 49 days gestation [3]. The regimen of mifepristone followed by misoprostol has become increasingly available and is now the gold standard for this indication. Studies have been ongoing to develop alternative regimens with these agents that are easier to administer, are cost effective, have low incidence of side effects and improve acceptability of the method. Misoprostol has also been studied as a single agent for elective termina- 
tion of pregnancy. Most published trials have evaluated a dose of 800 ug misoprostol administered every $24 \mathrm{hr}$, in gestations up to 70 days duration with a reported success rates of $85 \%$ - 93\% (Carbonell et al., 1997a,b; et al. Esteve et al., 1999; Jain et al., 1999; Bugallo et al., 2000; Ngai et al., 2000; Jain et al., 2001) [4-10]. It is easier to use, provide more rapid pregnancy termination and is less expensive compared with the mifepristone and misoprostol combination regimen. Moreover it can be used in areas where mifepristone is unavailable or is difficult to obtain. Therefore, this study was designed to compare mifepristone and vaginal misoprostol to vaginal misoprostol alone for elective termination of early pregnancy.

\section{MATERIAL AND METHODS}

A total of 200 healthy women desiring termination of pregnancies $<56$ days were enrolled after a review of subject's history, complete physical examination, pelvic ultrasound to confirm gestational age (if required), and documentation of informed consent. Haemoglobin levels and Rh type were also done at enrolment visit (day 1).

Exclusion criteria included any evidence of threatened or spontaneous abortion as defined by prior episodes of uterine bleeding or the presence of cervical dilatation.

Other exclusion criteria included anaemia (haemoglobin $<8 \mathrm{~g} / \mathrm{dl}$ ), bleeding disorders, patient on long term systemic steroids, patients on anticoagulants, chronic adrenal failure, severe renal, respiratory or liver disease, uncontrolled hypertension, glaucoma,cardiovascular or cerebrovascular disease, uncontrolled seizure disorder presence of uterine leiomyomata or any known allergy or contraindication to the study medications, lack of access to emergency care.

Subjects were randomized to one of two groups. Women assigned to group I received tab mifepristone $200 \mathrm{mg}$ on day 1 , and women in group 2 received placebo medication.

Subjects were told to record uterine bleeding episodes, side effects, and any medications used on each day until final visit.

On day 3 , the subjects returned and four $200 \mathrm{ug}$ tablets (800 ug) of misoprostol were placed vaginally under direct visualization with a speculum, followed by placement of $2 \mathrm{ml}$ of normal saline to moisten the tablets. Prophylactic doses of loperamide $(4 \mathrm{mg})$ and acetaminophen $(1000 \mathrm{mg}$ ) were administered to each subject. Subjects remained supine for 30 minutes before going home. Those subjects who had no bleeding on day 4 were given the option to have surgical evacuation to terminate pregnancy. All subjects were contacted on day 8 by telephone to evaluate symptoms and all subjects were scheduled to return on study day 15 for a follow up visit. At that time, subjects underwent a repeat pelvic ultrasound and haemoglobin measurement. The subjects also returned their symptom dairy detailing uterine bleeding patterns (none, spotting, moderate, heavy), and incidence of pain, nausea, vomiting or diarrhoea for each day during the study protocol. All subjects returning for final visit completed a questionaire concerning acceptability of the method. The pain perceived by patient was assessed on a visual analogue scale (VAS).

Abortion failure was defined as a need for evacuation of the uterus by a surgical technique for any reason, including the presence of a persistent gestational sac sonographically, excessive or prolonged bleeding, incomplete abortion, or subject's request. Surgical evacuation was performed by electric or manual vaccum aspiration after informed consent.

\section{OBSERVATIONS}

Demographic profile, successful abortion and side effects are shown in Tables 1, 2 and $\mathbf{3}$ respectively.

No severe complications occurred in any subjects, including blood transfusion, emergent surgery for haemmorhage, or sepsis. There was no statistically significant difference in mean haemoglobin change between 2 regimens studied. A total only 2 subjects in group I and 4 subjects in group II had a decrease of $>2 \mathrm{gm} / \mathrm{dl}$ in haemoglobin over the course of the study ( $p$ value not significant). Acceptability of the regimens was estimated by exit questionnaires. There was no statistically significant high incidence of side effects, an overwhelming majority of subjects still reported the method to be tolerable or very tolerable. The use of additional medication to treat any of these side effects was rare among the study population $(3 \%)$.

\section{DISCUSSION}

In the last decade, medical abortion has emerged as a realistic alternative to surgical abortion. Medical abortion has been described as a safe and natural method by the patients who have had an experience with it. This study was conducted on 200 pregnant women of period of gestation $\leq 56$ days to study the efficacy and side effects of the drug protocol.

Table 1. Demographic characteristics of the study population.

\begin{tabular}{lccc}
\hline Characteristic & $\begin{array}{c}\text { Gr I:mifepristone } \\
\text { and misoprostol } \\
(\mathrm{n}=100)\end{array}$ & $\begin{array}{c}\text { Gr II: placebo } \\
\text { and misoprostol } \\
(\mathrm{n}=100)\end{array}$ & P value \\
\hline Age (yr) & $29.39+/-5.10$ & $29.34+/-4.80$ & $\mathrm{NS}$ \\
Parity & $1.95+/-0.80$ & $1.85+/-0.75$ & $\mathrm{NS}$ \\
\hline $\begin{array}{l}\text { Previous abortions } \\
0\end{array}$ & 72 & 76 & $\mathrm{NS}$ \\
1 & 28 & 24 & \\
2 or more & 0 & 0 & \\
\hline
\end{tabular}

Gestational age (days) $45.06+/-6.19 \quad 44.25+/-5.89 \quad$ NS 
In the present study the mean age of the patients was $29.39+/-5.10$ in group I and $29.34+/-4.80$ in group II. Majority of women were in 21 - 30 years age group. The mean age of patients was 22.3 years in the study by Scaff et al and 26 years in both buccal and vaginal groups in the study by Middleton et al. [11,12]. In the present study $54.5 \%$ patients were from urban background with remaining $35.5 \%$ coming from rural background. Mean parity was $1.95+/-0.80$ in group I and $1.85+/-0.75$ in group II. Maximum number of patients $(45.5 \%)$ had previous two live births. This trend probably indicates that women opting for an abortion have completed their family and want to limit their family size. The mean period of gestation was $45.06+/-6.19$ in group I with the range of $35-56$ and $44.25+/-5.89$ in group II with the range of 33 - 56 days. In the present study mean time interval between onset of bleeding and misoprostol administration was $5.02+/-2.13$ hours in group I and $6.84+/-3.92$ in group II. The mean interval between onset of bleeding and misoprostol administration was significantly earlier in group I than group II ( $\mathrm{p}$ value $<0.005$ ). 96 percent of the patients reported bleeding within 24 hours of misoprostol administration. 7 patients in group II did not bleed at all.

Table 3 shows incidence of side effects in the two groups on day $1-2$ and on day $>3$ of the study and whether any medication was used by the subjects to treat nausea, vomiting or diarrhea. No severe complications occurred in any subjects, including blood transfusion, emergent surgery for haemmorhage, or sepsis. In the study by El Rafaey et al. the incidence of side effects in oral versus vaginal groups was nausea $70 \%$ vs $60 \%$, vomiting $44 \%$ vs $31 \%$, diarrhea $36 \%$ vs $18 \%$, dizziness $41 \%$ vs $44 \%$ and headache $22 \%$ vs $21 \%$. The incidence of gastrointestinal side effects were higher with oral than vaginal route [13].

In the present study the rate of complete abortion was $96 \%$ in group I and 79\% in group II. There were 4 cases of incomplete abortion in group I and 13 cases of incomplete abortion, 3 ongoing pregnancies and 5 missed abortions in group II. These were evacuated surgically after 15 days. All the 4 cases of group I and 12 out of 21 cases in group II had period of gestation 50 - 56 days showing that medical abortion may be less efficacious if period of gestation is $>49$ days. Also, there was insignificant difference in success of abortion between group I and group II if period of gestation is between 50 - 56 days, this may be due to less no of subjects having period of gestation 50 - 56 days. The overall acceptance rate of medical abortion over surgical abortion was $100 \%$, though the

Table 2. Successful abortion rate by gestational age.

\begin{tabular}{llll}
\hline Gestational age & Gr I mifepristone and misoprostol & Gr II placebo and misoprostol & P \\
\hline$<49$ days gestation & $75 / 75(100 \%)$ & $66 / 75(88 \%)$ & $<0.001$ \\
$50-56$ days gestation & $21 / 25(84 \%)$ & $13 / 25(52 \%)$ & $>0.05$ \\
\hline
\end{tabular}

Table 3. Incidence of self-reported symptoms by treatment group.

\begin{tabular}{|c|c|c|c|}
\hline Symptoms & $\begin{array}{l}\text { Group I mifepristone and } \\
\text { misoprostol }\end{array}$ & $\begin{array}{l}\text { Group II placebo and } \\
\text { misoprostol }\end{array}$ & $P$ value \\
\hline \multicolumn{4}{|l|}{ Reported on day 1 - $2(\%)$} \\
\hline Cramping & 3.0 & 3.0 & \\
\hline Nausea & 32.0 & 28.0 & NS \\
\hline Vomiting & 22.6 & 5.1 & $<0.001$ \\
\hline Diarrhoea & 7.4 & 2.5 & $<0.05$ \\
\hline Fever/chills & 27.1 & 12.4 & 0.05 \\
\hline \multicolumn{4}{|l|}{ Reported on or after study day $3(\%)$} \\
\hline Nausea & 44.1 & 46.0 & NS \\
\hline Vomiting & 27.2 & 21.7 & NS \\
\hline Diarrhoea & 16.4 & 11.4 & NS \\
\hline Fever/chills & 60.9 & 71.0 & NS \\
\hline $\begin{array}{l}\text { Subjects who needed medication for } \\
\text { nausea, vomiting or diarrhoea }(\%)\end{array}$ & 1 & 3.2 & NS \\
\hline $\begin{array}{l}\text { Maximum temp of subjects reporting } \\
\text { fever/chills (F) }\end{array}$ & $100.0+/-1.67$ & $99.9+/-1.35$ & NS \\
\hline
\end{tabular}


overall acceptability to route was $73 \%$. In the developing countries like ours, cost is an important factor and as mifepristone is an expensive drug it can not be afforded by poor patients. In such situations, misoprostol alone regimen offers advantage in terms of its lower cost.

This study and others (Arvidsson C 2005, Fjerstad M 2009) confirm that subjects in medical abortion trials find the procedure highly acceptable [14,15]. Mifepristone appears to have a small but clinically and statistically significant effect on abortion success. Misoprostol as a single agent for pregnancy termination is not a very good method for medical termination of early pregnancy. Further investigation is needed to evaluate the efficacy of misoprostol alone for earlier gestations $(<49$ days or even $<42$ days). Misoprostol as a single agent for pregnancy termination remains of value in clinical situations where mifepristone is unavailable or contraindicated, especially due to much lower cost of misoprostol compared with mifepristone.

\section{REFERENCES}

[1] World Health Organization (2004) Unsafe abortion- global and regional estimates of the incidence of unsafe abortion and associated mortality in 2000. 4th Edition, WHO, 82.

[2] Avrech, O.M., Golan, A. and Weinraub, Z. (1991) Mefipristone alone or in combination with an analogue for termination of early pregnancy: A review. Fertility and Sterility, 56, 385-393.

[3] Jain, J.K., Dutton, C. and Harwood, B. (2002) A prospective randomized, double blinded, placebo-controlled trial comparing, mifepristone and vaginal misoprostol to vaginal misoprostol alone for elective termination of early pregnancy. Human Reproduction, 17, 1477-1482. doi:10.1093/humrep/17.6.1477

[4] Carbonell, J.L., Varela, L. and Velazco, A. (1997) The use of misoprostol for termination of early pregnancy. Contraception, 55, 165-168. doi:10.1016/S0010-7824(97)00020-6

[5] Carbonell, J.L., Varela, L. and Velazco, A. (1997) The use of misoprostol for abortion at $<9$ weeks gestation. European Journal of Contraception and Reproductive Health Care, 2, 181-185. doi:10.3109/13625189709167474

[6] Bugalho, A., Mocumbi, S., Faundes, A. and David, E.
(2000) Termination of pregnancies of $<6$ weeks gestation with a single dose of $800 \mathrm{ug}$ of vaginal misoprostol. Contraception, 61, 47-50. doi:10.1016/S0010-7824(99)00116-X

[7] Esteve, J.L., Varela, L. and Velazco, A. (1999) Early abortion with $800 \mathrm{ug}$ of misoprostol by vagonal route. Contraception, 59, 219-225. doi:10.1016/S0010-7824(99)00032-3

[8] Jain, J.K., Meckstroth, K.R. and Mishell, D.R. Jr. (1999) Early pregnancy termination with intravaginally administered sodium chloride solution-moistened misoprostol tablets: Historical comparison with mifepristone and oral misoprostol. American Journal of Obstetrics \& Gynecology, 181, 1386-1391. doi:10.1016/S0002-9378(99)70380-7

[9] Nagai, S.W., Tang, O.S. and Chan, Y.M. (2000) Vaginal misoprostol alone for medical abortion up to 9 weeks of gestation: Efficacy and acceptability. Human Reproduction, 15, 1159-1162. doi:10.1093/humrep/15.5.1159

[10] Jain, J.K., Harwood, B., Meckstroth, K.R. and Mishell, D.R. Jr. (2001) Early pregnancy termination with vaginal misoprostol combined with loperamide and acetaminophen prophylaxis. Contraception, 63, 217-221. doi:10.1016/S0010-7824(01)00193-7

[11] Schaff, E.A., DiCenzo, R. and Fielding, S.L. (2005) Comparison of misoprostol plasma concentrations following buccal and sublingual administration. Contraception, 71, 22-25. doi:10.1016/j.contraception.2004.06.014

[12] Middleton, T., Schaff, E. and Fielding, S.L. (2005) Randomized trial of mifepristone and buccal or vaginal misoprostol for abortion through 56 days of last menstrual period. Contraception, 72, 328-332. doi:10.1016/j.contraception.2005.05.017

[13] El Refaey, H., Rajasekar, D. and Abdalla, M. (1995) Induction of abortion with mifepristone (RU 486) and oral or vaginal misoprostol. The New England Journal of Medicine, 332, 983-987. doi:10.1056/NEJM199504133321502

[14] Arvidsson, C., Hellborg, M. and Gemzell-Danielsson, K. (2005) Preference and acceptability of oral versus vaginal administration of misoprostol in medical abortion with mifepristone. European Journal of Obstetrics \& Gynecology and Reproductive Biology, 123, 87-91. doi:10.1016/j.ejogrb.2005.02.019

[15] Fjerstad, M., Sivin, I. and Lichtenberg, E.S. (2009) Effectiveness of medical abortion with mifepristone and buccal misoprostol through 59 gestational days. Contraception, 80, 282-286. doi:10.1016/i.contraception.2009.03.010 